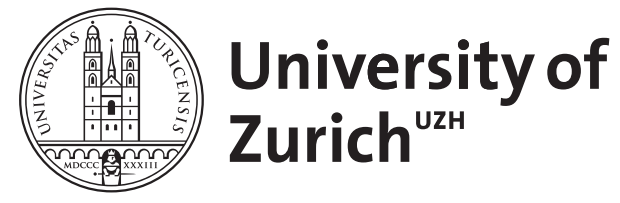

\title{
Schwieriger präklinischer Atemweg und Bergung an der Rettungswinde
}

\author{
Stein, P ; Meyer, J L ; Mariotti, S ; Spahn, D R ; Albrecht, R
}

DOI: https://doi.org/10.1055/s-0034-1387585

Posted at the Zurich Open Repository and Archive, University of Zurich ZORA URL: https://doi.org/10.5167/uzh-113629

Journal Article

Accepted Version

Originally published at:

Stein, P; Meyer, J L; Mariotti, S; Spahn, D R; Albrecht, R (2015). Schwieriger präklinischer Atemweg und Bergung an der Rettungswinde. Der Notarzt, 31(03):129-133.

DOI: https://doi.org/10.1055/s-0034-1387585 
Schwieriger präklinischer Atemweg und Bergung an der Rettungswinde

Difficult prehospital airway and hoist rescue operation

Autoren:

P. Stein ${ }^{1,2}$, J.L. Meyer ${ }^{1,2}$, S. Mariotti ${ }^{3}$, D.R. Spahn ${ }^{1}$, R. Albrecht ${ }^{2}$

Institute:

${ }^{1}$ Institut für Anästhesiologie, Universitätsspital Zürich, Zürich, Schweiz

${ }^{2}$ Schweizerische Rettungsflugwacht (Rega), Zürich-Flughafen, Schweiz

${ }^{3}$ Rettungsdienst Schutz \& Rettung der Stadt Zürich, Zürich, Schweiz

Schlüsselwörter:

Windenrettung

Larynxtubus

Schwieriger Atemweg

Halswirbelsäulenverletzung

Keywords:

Hoist rescue operation

Laryngeal tube

Difficult airway

Cervical spine injury

Korrespondenzadresse:

Philipp Stein

Institut für Anästhesiologie

Universitätsspital Zürich

Rämistrasse 100

8091 Zürich - Schweiz

philipp.stein@usz.ch 
Die schriftliche Einwilligung des Patienten zur Publikation liegt vor.

Zusammenfassung: Vorstellung der Kasuistik eines 60 jährigen Patienten mit Verletzung durch einen schweren Gegenstand am zervikothorakalen Übergang. Bei progredienter Dyspnoe in Folge einer entstandenen Paraplegie erfolgte die Intubation mittels Larynxtubus bei schwierigem Atemweg. Bergungstechnisch wurde die Indikation zur Windenbergung des Patienten mittels Rettungshelikopter im unwegsamen Baustellengelände gestellt. Diskussion der folgenden Punkte: Präklinischer schwieriger Atemweg und Windenbergung eines beatmeten Patienten

Abstract: Case report of a 60 -year-old patient with a cervicothoracic spine injury secondary to a strike by a heavy object. Because of progressive difficulties to breathe due to paraplegia, the patient was intubated with a laryngeal tube in the context of a difficult airway. The transport of the patient was facilitated by a rescue helicopter hoist operation. The following subjects will be discussed: Prehospital difficult airway and hoist rescue operation with a ventilated patient.

\section{Epikrise:}

Ein männlicher 60 jähriger Bauarbeiter wurde im Februar 2014 nachmittags von einem ca. zwei Tonnen schweren Betonkübel an Halswirbelsäule und Hinterkopf getroffen. Bei Eintreffen von Rettungsdienst und Notarzt um 15:00 Uhr wurde der Patient in einer sitzenden aber zusammengesunkenen Position vorgefunden. Das erste Assessment ergab:

A: Atemweg frei -> Anlage Halswirbelsäulenfixation.

B: Spontanatmung, SpO2 99\% ohne zusätzlichen Sauerstoff, auskultatorisch vesikuläres Atemgeräusch beidseits, subjektiv klagte der Patient über massive Dyspnoe bei Einsatz der zervikalen Atemhilfsmuskulatur.

C: Sinusrhythmus, Herzfrequenz 90/Min, nicht invasive Blutdruckmessung 180/110mmHg. Anlage venöser Zugänge an den Handrücken G18 links und G16 rechts.

D: GCS 15, Pupillen isokor und lichtreagibel, periphere Sensomotorik: inkomplette Tetraplegie. Beim Traumacheck äußerte der Patient Schmerzen im Bereich der Brustwirbelsäule. Palpatorisch war keine Stufenbildung der Wirbelsäule feststellbar.

E: Sagittale frontoparietale Skalpierungsverletzung, Baustellengelände mit schwierigem Zugang.

In der Kurzanamnese wurden folgende zusätzlichen Parameter erhoben: keine bekannten Allergien, keine Medikamenteneinnahme oder Vorerkrankungen, letzte Mahlzeit um etwa 12:30 Uhr.

Zur schnellen Bergung des schwer verletzen Patienten im unwegsamen und abschüssigen Baustellengelände wurde die Schweizerische Rettungsflugwacht (Rega) alarmiert. So ging um 15:12 folgende Einsatzmeldung auf der Helikopter Basis in Dübendorf (Rega 1) ein: Windenbergung Baustelle, männlich, Kopfplatzwunde, GCS 15. Die Crew bereitete sich in der fünf minütigen Bereitschaftszeit für den Einsatz vor, die Notärztin legte den für den Windeneinsatz benötigten Klettergurt an, der Jungfrau-Bergesack (Mod. Rega, Tyromont, Innsbruck / Österreich) wurde für den Einsatz bereit gemacht und der Paramedic, der bei Windeneinsätzen mit einer speziellen Weste im Eurocopter EC 145 gesichert ist, legte sein 
Equipment an. Um 15:20 schwebte der Hubschrauber über dem Einsatzort. Die Baustelle lag am Hang und für den Windeneinsatz war nur wenig Platz vorhanden. Das Wetter war zum aktuellen Zeitpunkt trocken, aber windig. Der Untergrund war nach einem vorangegangenen Regenschauer nass und rutschig.

Ablauf und Kommunikation bei der Windenaktion zwischen Arzt, Pilot und Windenoperator (Paramedic) sind in einer "standard operating procedure“ (SOP) festgelegt. Der Pilot bekommt vom Paramedic die finalen Richtungsanweisungen, nur er hat direkten Sichtkontakt zum angestrebten Landeplatz und zur Notärztin. Die Notärztin gibt etwa 4 Meter über dem Boden ein Handzeichen, woraufhin die Windengeschwindigkeit verlangsamt wird, um den Bodenkontakt zu vereinfachen. Um 15:27 traf die Notärztin mitsamt Jungfrau-Bergesack beim Patienten ein.

Währenddessen suchte sich der Pilot einen Zwischenlandeplatz auf einer Wiese oberhalb der Baustelle. Dort wurde das Tragesystem ausgeladen, das Pack Rack (Tisch, auf dem Monitor und Beatmungsgerät befestigt sind) von der Trage getrennt, um später den Patienten direkt auf der Trage absetzen zu können.

Durch den bodengebundenen Notarzt vor Ort war in der Zwischenzeit bei progredienter Tetraplegie und zunehmender Dyspnoe die Indikation zur Intubation gestellt worden. Der Patient wurde in unverändert sitzender Position präoxygeniert. Die Anästhesieeinleitung erfolgte im Sinne einer „rapid sequence induction“ mit Fentanyl, Propofol und Succinylcholin in üblichen Dosierungen. Nach der Medikamentengabe wurde der Patient achsengerecht auf ein zuvor unter das Gesäß des Patienten gelegtes Rettungsbrett umgelagert. Bei der direkten Laryngoskopie zeigte sich bei "Inlinestabilisierung“ ein Intubationssitus nach Cormack und Lehane Grad 4, eine konventionelle Intubation war somit nicht möglich. Ein Larynxtubus (LTS-D, VBM Medizintechnik, Sulz a.N., Deutschland) ließ sich jedoch problemlos platzieren.

Bei der Übernahme durch die Notärztin des RTH Rega 1 war der Atemweg mittels Larynxtubus gesichert und es erfolgte die manuelle Beatmung des Patienten mittels Ambubeutel. Ein Atemgeräusch war beidseits auskultierbar, ein $\mathrm{CO}_{2}$ von $50 \mathrm{mmHg}$ und eine periphere Sauerstoffsättigung von $99 \%$ waren messbar. Der Patient war mit einem Blutdruck von 90/60 mmHg mittlerweile leicht hypotensiv, mit einer Herzfrequenz von 75/Min. aber unverändert kreislaufstabil. Die Sedierung wurde mit Midazolam aufrechterhalten, die Relaxation mit Rocuronium weitergeführt.

Es erfolgte die Umlagerung des Patienten vom Rettungsbrett in den Jungfrau-Bergesack. Das Monitoring des Rettungsdienstes wurde aus Zeitgründen komplett übernommen, die manuelle Beatmung fortgesetzt. Monitor und Beatmungsgerät hätten sonst per Funk angefordert und anschließend eingeflogen werden müssen.

Der Akku des Monitormoduls wurde auf dem Bauch des Patienten im Jungfrau-Bergesack verstaut und die Sauerstoffflasche zwischen den Beinen des Patienten fixiert. Der Monitor selbst wurde zur Überwachung des Patienten außen am Jungfrau-Bergesack befestigt. Die Anzeige war jedoch durch die Sonneneinstrahlung nur schwierig ablesbar. Per Funk wurde nun der Hubschrauber für die Windenbergung angefordert. Die Stranggehänge des Jungfrau-Bergesackes waren bereits mittels Rettertau (zwei Karabiner mit Dreifachverschluss verbunden durch ein kurzes Stück Seil) mit der Notärztin verbunden. Bei Ankunft des Hubschraubers wurde dessen Windenhaken eingehängt, bevor nach einer abschließenden Sichtkontrolle das Zeichen zum Beginn der Windenaktion gegeben werden 
konnte. Unter manueller Beatmung begann so um 15:52 die Windenbergung. Patient und Notärztin wurden bis an die Kufe des Hubschraubers hochgezogen und dort durch die Notärztin während des Fluges stabilisiert (Abb. 4). Über dem Zwischenlandeplatz schwebend, wurden Notärztin und Patient per Winde wieder abgesetzt, kurz danach landete der Hubschrauber in unmittelbarer Nähe von Patient und vorbereitetem Tragesystem. Danach erfolgte das Einladen des Patienten in den RTH.

Im folgenden „Reassessment“ fiel auf, dass der Larynxtubus unter maschineller Beatmung ein Luftleck aufwies. Die restlichen Vitalparameter waren weiterhin stabil. Daher erfolgte um 16:05 der erneute Start des RTH und der Abflug Richtung Universitätsspital Zürich mit dortiger Landung um 16:13 und anschließender Übergabe des Patienten im Schockraum um $16: 15$.

Bei nun deutlich undichtem Larynxtubus erfolgte die sofortige problemlose fiberoptische Intubation des Patienten. Dabei wurde die Fiberoptik bei fortbestehender HWS Fixation am entblockten Larynxtubus vorbei endotracheal eingeführt und darüber ein Endotrachealtubus geschoben. Nach Bestätigung der korrekten Tubuslage mittels Fiberoptik und Kapnographie wurde der Larynxtubus entfernt.

Während der anschließenden Schockraumdiagnostik wurden folgende Befunde erhoben (Abb. 1):

- Luxationsfraktur C6/7 mit inkompletter Tetraplegie unterhalb C6

- Berstungsfrakturen HWK 7 und BWK 1

- Inkomplette Berstungsfraktur BWK 3

- Vordere Atlasbogenfraktur rechts (Gehweiler IV)

- Leichtes Schädel-Hirn-Trauma mit Skalpierungsverletzung

- Extraglenoidale Skapulatrümmerfraktur links

Noch am Unfalltag wurden eine dekompressive Laminektomie C 5-7, sowie eine dorsale Spondylodese HWK 3-BWK 5 durchgeführt. Im Verlauf erfolgten eine Korpektomie des HWK 7 und BWK 1, sowie eine Dekompression von C 7 und eine ventrale "Cagespondylodese“ HWK 6-BWK 2.

Bei der zeitgleich durchgeführten offenen Tracheotomie fiel bei der konventionellen Laryngoskopie bei freigegebener HWS und unter innerklinischen Bedingungen ein Intubationssitus nach Cormack und Lehane Grad 3 auf.

Eine Woche nach dem Unfall wurde der Patient zur weiteren Therapie in ein Paraplegikerzentrum verlegt. Das neurologische Defizit bestand zu diesem Zeitpunkt sensomotorisch sub C6. Motorisch bestand ein Kraftgrad der Schulterabduktion beidseits von M3, der Ellenbogenflexion rechts von M3, links von M2, sowie der Hände und unteren Extremitäten von $\mathrm{M} 0$.

\section{Diskussion Atemwegssicherung:}

Die präklinische Atemwegssicherung stellt für den Notarzt eine große Herausforderung dar. Einerseits ist die Rate an schwierigen Intubationen häufiger als innerklinisch [1], andererseits erschweren die äußeren Umstände, wie Umgebung, Verletzungsmuster und eingeschränkte (Intubations-) Materialressourcen den Vorgang zusätzlich. 
Der Goldstandard für die Atemwegssicherung stellt auch gemäß der S3 Leitlinie „Polytrauma“ zur Intubation [2] die endotracheale Intubation dar. Dieser Goldstandard ist aber nicht immer anwendbar und kann in der Hand des Unerfahrenen eine Gefährdung des Patienten darstellen [3], wenn nicht die Algorithmen zur Erkennung der sicheren endotrachealen Intubation abgearbeitet werden und bei Versagen nicht auf Alternativen ausgewichen wird.

Eine dieser Alternativen stellt der Larynxtubus dar. Dieser wird „blind“ oral eingeführt. Ein großer pharyngealer und ein kleiner ösophagealer Cuff dichten den Luftstrom nach oben und unten $a b$ und bieten einen gewissen Aspirationsschutz (Abb. 2). Die Luftöffnung liegt im Bereich der Glottis, die Spitze des Larynxtubus liegt im Ösophagus (Abb. 3). Über die ösophageale Öffnung kann eine Magensonde eingeführt werden (Modell LTS-D). Diese supraglottische Atemwegshilfe findet insbesondere präklinisch, aber auch innerklinisch zunehmend Verwendung zur Etablierung eines supraglottischen Atemweges [4]. Darüber hinaus werden weitere supraglottische Atemwegshilfen wie die Larynxmaske und die Intubationslarynxmaske [5] erfolgreich angewandt.

Videolaryngoskope werden immer mehr auch präklinisch angewendet [6]. Allerdings werden diese aktuell bei der Rega nicht eingesetzt, da die schweizweit eingesetzten Notärzte innerklinisch nicht flächendeckend einheitlich diese Hilfsmittel anwenden. Als Alternative zum nicht erfolgreichen Standard (konventionelle endotracheale Intubation) muss ein bekanntes Hilfsmittel eingesetzt werden, da auch die Videolaryngoskopie eine gewisse Lernkurve aufweist und mögliche Atemwegsschädigungen bei der Platzierung des Tubus auftreten können [7]. Videolaryngoskope werden für den zukünftigen Einsatz bei der Rega evaluiert, um ein weiteres Hilfsmittel bei der nicht selten auftretenden schwierigen präklinischen Intubation zu besitzen und den Goldstandard (endotracheale Intubation) mit größerer Wahrscheinlichkeit zu ermöglichen. Die adäquate innerklinische Verbreitung und Schulung des vorgehaltenen Equipments muss im Vorfeld sichergestellt werden. Ebenso müssen die möglichen Komplikationen bei der Anwendung des Larynxtubus bekannt sein und diesen Rechnung getragen werden [8].

Alle 16 Rettungshubschrauber der Rega sind einheitlich ausgerüstet. Vorhanden sind Endotrachealtuben von $2.5-8 \mathrm{~mm}$ Innendurchmesser. Zwei unterschiedlich große Laryngoskopgriffe und 5 unterschiedliche Spatel stehen für die Laryngoskopie zur Verfügung. Zur alternativen Atemwegssicherung werden 3 Kinderlarynxmasken Größe 1.52.5 (LMA unique, LMA GmbH, 53175 Bonn, Deutschland), 3 Intubationslarynxmasken Größe 3-5 (LMA Fastrach, LMA GmbH, 53175 Bonn, Deutschland), 2 Stilette (METTS CH 8, VBM Medizintechnik, Sulz a.N., Deutschland, Portex CH 15, Smiths medical int. Ltd., Hyte, Kent, UK) und 2 Quicktrach (VBM Medizintechnik, Sulz a.N., Deutschland) Systeme mitgeführt. Die Notwendigkeit der Verwendung alternativer Atemwegshilfen ist selten (interne Rega Daten).

Diskussion Rettungsablauf:

Die Einsatzmeldung über einen bewusstseinsklaren Patienten beeinflusste die Vorbereitungen und den eigentlichen Rettungsablauf maßgeblich.

Da das Universitätsspital in Zürich zum Teil die Notärzte sowohl für den Luftrettungsdienst der Rega, als auch für den bodengebundenen Rettungsdienst Schutz \& Rettung der Stadt Zürich stellt, war sowohl das ersteintreffende Team am Einsatzort, als auch das verwendete Material der Helikopternotärztin bekannt. Damit ließ sich auch das vorhandene Monitoring 
problemlos übernehmen, zusätzliche Probleme und Zeitverlust konnten so vermieden werden. Das erneute Einfliegen von zusätzlichem Material wie Monitoring, Beatmungsgerät und zusätzlichen Atemwegshilfen per Winde hätte zu einer Verzögerung der Rettung geführt. Zudem ist eine sichere Befestigung von weiterem Material schwierig und bei der folgenden Windenaktion aufgrund von Größe und Gewicht weder sinnvoll noch empfehlenswert.

Eine Windenbergung bei wachen Patienten erfolgt in der Regel ohne Monitoring. Der beatmete Patient benötigt als Standard bei der Rega als minimales Monitoring eine periphere Sauerstoffsättigung und eine Kapnographie. In der Regel sind im Rahmen der Atemwegssicherung auch Blutdruckmessung und EKG-Überwachung installiert. Allerdings ist die technische Überwachungsmöglichkeit an der Winde deutlich eingeschränkt. Die Geräte können sich außerhalb des Sichtbereiches befinden und das Sonnenlicht kann das Ablesen unmöglich machen. Eine Kompression des Beatmungsbeutels und die Unmöglichkeit der Beatmung durch den „Downwash“ des Helikopters sind beschrieben [9].

Gemäß aktuellen Recherchen ist dies der erste beschriebene Fall eines mittels Larynxtubus beatmeten Patienten an der Rettungswinde. Der Larynxtubus gehört nicht zur medizinischen Ausstattung der Rega, ist jedoch bei Einsätzen mit bodengebundenen Partnerorganisationen durchaus regelhaft anzutreffen. Der Larynxtubus weist im Vergleich zur LMA classic ${ }^{\mathrm{TM}}$ und auch zur LMA Fastrach ${ }^{\mathrm{TM}}$ einen deutlich höheren Atemwegs-Leckagedruck auf [10]. In anderen Untersuchungen wiesen der Larynxtubus LTS II und die Intubationslarynxmaske LMA-ProSeal vergleichbare Erfolgsraten beim Platzieren auf [11]. Das präklinische Atemwegsmanagement der Rega wird fortlaufend intern evaluiert und den internationalen Guidelines angepasst. Gemäß internen Auswertungen existiert keine dringliche Notwendigkeit, das Atemwegsequipment um einen Larynxtubus zu erweitern. Dies wird stetig neu evaluiert und gegebenenfalls dem Bedarf bzw. der sich verändernden Rettungsdienstlandschaft angepasst.

Der Transport von beatmeten Patienten an der Winde stellt eine große Ausnahme dar [12]. Es gibt hierfür keine SOP. Das Monitoring ist minimal und es muss ausgeprägten Wert auf eine klinische Beobachtung des zuvor etablierten Atemwegs gelegt werden. Mögliche geeignete automatische Beatmungsmethoden an der Winde wurden beschrieben [13]. Es gibt nur wenig Evidenz über physiologische Veränderungen der Lungenfunktion an der Rettungswinde [14].

Der Zeitraum dieser reduzierten Überwachung soll so kurz wie möglich gehalten werden, um umgehend zum Standardmonitoring zurückzukehren. Der Transport von beatmeten Patienten an der Rettungswinde soll weiterhin die absolute Ausnahme darstellen.

Häufig ist im unwegsamen oder alpinen Gelände am Unfallort weder Rettungsdienst noch anderes Hilfspersonal vorhanden. Hier muss sinnvoll abgewogen werden, ob überhaupt notärztliche Maßnahmen am Unfallort durchgeführt werden können und sollen. Selbst bei klassischen Intubationsindikationen wie Schädelhirntrauma und Vigilanzminderung GCS < 9 , muss der Bergung (mittels Winde) häufig Vorrang vor invasiven notärztlichen Tätigkeiten gegeben werden. Diese können dann gegebenenfalls erst am Zwischenlandeplatz mit Unterstützung des Paramedic erfolgen.

\section{Würden wir es wieder so machen?}

Für den Windeneinsatz bei beatmeten Patienten gibt es wie erwähnt bei der Rega keine SOP, die Bergung des Patienten wurde nach bestem Wissen und Gewissen mit den vor Ort zur Verfügung stehenden Mitteln durchgeführt. Ob sich ein beatmeter Patient für einen Windeneinsatz eignet, hängt sicherlich auch von seinen Begleitverletzungen ab. Der hier 
geborgene Patient war trotz seiner hohen Tetraplegie kreislaufstabil. Der Einsatz eines Rettungshelikopters im Baustellenbereich ist zudem häufig aufgrund der Staubentwicklung und möglicher ungesicherter Bauteile schwierig bis unmöglich.

Diesen Umständen muss bei der Risikoanalyse vor jeder geplanten Aktion erneut Rechnung getragen werden.

Interessenskonflikt:

Alle Autoren geben an, dass kein Interessenskonflikt besteht. 
Literatur:

1. Breckwoldt J, Klemstein S, Brunne B, Schnitzer L, Mochmann H-C, Arntz H-R. Difficult prehospital endotracheal intubation - predisposing factors in a physician based EMS.

Resuscitation. Elsevier; 2011;82:1519-24.

2. Bernhard M, Matthes G, Kanz KG et al. [Emergency anesthesia, airway management and ventilation in major trauma. Background and key messages of the interdisciplinary S3 guidelines for major trauma patients]. Anaesthesist. 2011;60:1027-40.

3. Stein P, Albrecht R, Spahn DR. Multiple trauma, resuscitation, and 15 minutes of esophageal intubation: survival without neurologic deficit. Am J Emerg Med.

2014;32:950.e1-2.

4. Schalk R, Meininger D, Ruesseler M, Oberndörfer D, Walcher F, Zacharowski K, et al. Emergency airway management in trauma patients using laryngeal tube suction. Prehosp Emerg Care. 2011;15:347-50.

5. Bosch J, de Nooij J, de Visser M et al. Prehospital use in emergency patients of a laryngeal mask airway by ambulance paramedics is a safe and effective alternative for endotracheal intubation. Emergency Medicine Journal. BMJ Publishing Group Ltd and the British Association for Accident \& Emergency Medicine; 2014;31:750-3.

6. Cavus E, Callies A, Doerges $V$ et al. The C-MAC videolaryngoscope for prehospital emergency intubation: a prospective, multicentre, observational study. Emerg Med J. 2011;28:650-3.

7. Amundson AW, Weingarten TN. Traumatic GlideScope $(\mathbb{B})$ video laryngoscopy resulting in perforation of the soft palate. Can J Anaesth. 2013;60:210-1.

8. Schalk R, Seeger FH, Mutlak $\mathrm{H}$ et al. Complications associated with the prehospital use of laryngeal tubes-A systematic analysis of risk factors and strategies for prevention.

Resuscitation. Elsevier; 2014;0.

9. Burns BJ, Edwards K, House T. Bag valve mask failure during HEMS intubated stretcher winch. Air Med. J. 2012;31:84-6.

10. Thee C, Serocki G, Doerges V et al. Laryngeal tube S II, laryngeal tube S disposable, Fastrach laryngeal mask and Fastrach laryngeal mask disposable during elective surgery: a randomized controlled comparison between reusable and disposable supraglottic airway devices. European Journal of Anaesthesiology. 2010;27:468-72.

11. Genzwuerker HV, Altmayer S, Hinkelbein J, Gernoth C, Viergutz T, Ocker H. Prospective randomized comparison of the new Laryngeal Tube Suction LTS II and the LMA-ProSeal for elective surgical interventions. Acta Anaesthesiologica Scandinavica. 2007;51:1373-7.

12. Carpenter J, Thomas F. A 10-year analysis of 214 HEMS backcountry hoist rescues. Air Med. J. 2013;32:98-101.

13. Lavon O, Hershko D, Barenboim E. The utility of flow-limited automated mechanical ventilation during airborne hoist rescue missions. Am J Emerg Med. 2010;28:523-6.

14. Murphy D, Garner A, Bishop R. Respiratory function in hoist rescue: comparing slings, stretcher, and rescue basket. Aviat Space Environ Med. 2011;82:123-7.

15. Cormack RS, Lehane J. Difficult tracheal intubation in obstetrics. Anaesthesia. 1984;39:1105-11. 
Bildunterschriften:

Abb. 1: Luxationsfrakturen der Halswirbelsäule. Zustand nach fiberoptischer endotrachealer Intubation im Schockraum.

Abb. 2: Korrekte Lage des Larynxtubus. Gelb eingefärbt ösophagealer und pharyngealer Cuff des Tubus mit Luftöffnung vor der Glottis (abgebildeter Patient nicht aus der Fallbeschreibung).

Abb. 3: Korrekte ösophageale Lage der Spitze des Larynxtubus dorsal des Larynx.

Abb. 4: Transport eines beatmeten Patienten an der Rettungswinde des Rega Eurocopter EC 145. Patient im Jungfrau-Bergesack, verbunden mit dem Notarzt über Rettertau.

Legende:

\begin{tabular}{|l|l|}
\hline Cormack und Lehane & Intubationssitus nach [15] \\
\hline Downwash & Abwind, erzeugt durch die Rotorblätter \\
\hline GCS & Glasgow coma scale \\
\hline LMA & Larynxmaske \\
\hline LTS & Larynxtubus \\
\hline Pack Rack & $\begin{array}{l}\text { Montagesystem für Monitor und } \\
\text { Beatmungsgerät auf der Rettungstrage }\end{array}$ \\
\hline Paramedic & $\begin{array}{l}\text { Rettungssanitäter bei der Rega. HEMS } \\
\text { (Helicopter Emergency Medical Services) } \\
\text { Crew Member }\end{array}$ \\
\hline Rega & Schweizerische Rettungsflugwacht \\
\hline RTH & Rettungstransporthubschrauber \\
\hline SOP & Standard operating procedure \\
\hline SRZ & $\begin{array}{l}\text { Rettungsdienst Schutz \& Rettung der Stadt } \\
\text { Zürich }\end{array}$ \\
\hline
\end{tabular}

